\title{
Correlations between Atmospheric Extinction and Meteorological Conditions
}

\author{
H.-G. Reimann ${ }^{1}$ V. Ossenkopf ${ }^{2}$ \\ ${ }^{1}$ Astrophysikalisches Institut und Universitäts-Sternwarte, Jena, Germany \\ ${ }^{2}$ Max-Planck-Gesellschaft, Arbeitsgruppe "Staub in Sternentstehungsgebieten" Jena, \\ Germany
}

\begin{abstract}
A retrospective investigation covering about twenty years of wide-band and intermediate-band photometry at Jena University observing station allows an analysis of variations of atmospheric extinction at different time scales. A comparison with an earlier investigation by Wempe (1947) permits to draw conclusions about the evolution of air pollution by aerosols over nearly half a century. Complementary weather observations obtained at the meteorological observing station of the Jena University allow to find correlations between the variation of extinction parameters and the changing weather situations. Parallel Mie calculations allow to interpret the variations of the measured extinction data in terms of size variations of water-rich aerosols.
\end{abstract}

\section{The observations}

Since 1968 atmospheric extinction observations have been performed at Großschwabhausen observing station of Jena University Observatory. Photoelectric photometry in three colour $U B V$, four colour $u v b y$, and six colour standard IHW comet filters have been carried out at the $90-\mathrm{cm}$ telescope. The atmospheric extinction coefficients and the zero points for each photometric night were determined from a sample of about 15 standard stars. The aerosol extinction coefficient $k_{\mathrm{D}}$ was determined by the correction for standard Rayleigh scattering and ozone absorption.

In general, an extinction law for aerosols of the form

$$
k_{\mathrm{D}}(\lambda)=2.5 \lg e^{-\beta \lambda^{-\alpha}} ;[\lambda / \mu \mathrm{m}],
$$

is assumed ( $\alpha$ - wavelength exponent, $\beta$ - turbidity factor).

The figure 1 contains the $\alpha-k_{\mathrm{D}}(V)$-diagram for all observations. We see that most of the observations follow one general law: $\alpha$ decreases with increasing $k_{\mathrm{D}}(V)$. All $\alpha$-values from nearly pure Rayleigh scattering up to completely grey light scattering occur. The dashed line represents the best fit for the data given by the exponential function $\alpha=4 \exp \left(-2.18 k_{\mathrm{D}}(V)\right)$. The observations at different time intervals are 
marked by different symbols. Full triangles: observations before 1970, open circles: observations 1975 - 1984, full squares: observations since 1985 .

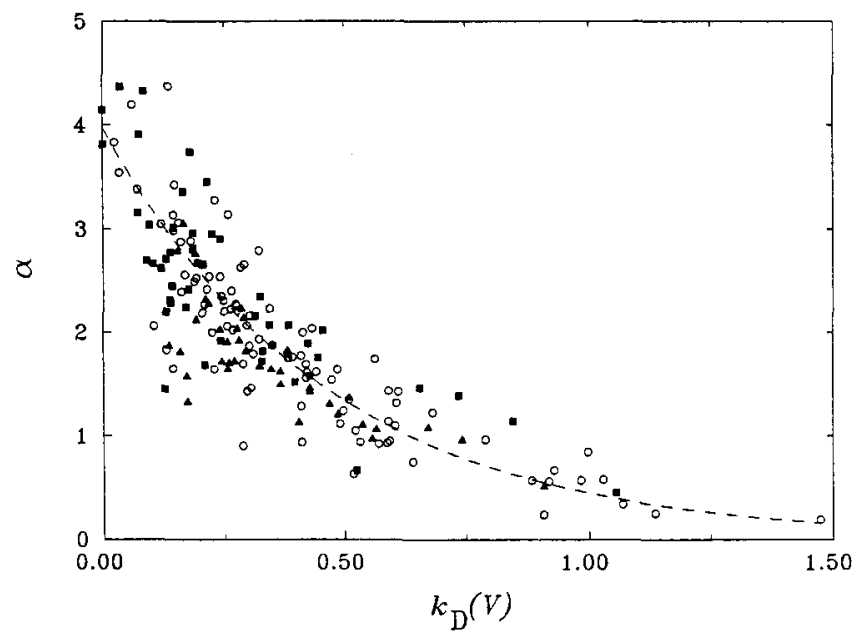

Figure 1 The $\alpha$ versus $k_{\mathrm{D}}(V)$ diagram.

\section{Simulation by Mie theory}

By means of Mie calculations we have tried to simulate the observed aerosol extinction. To characterize the models of typical aerosol distributions we used standard parameters as given by Koepke and Hess (1988) and optical constants from Twitty and Weinmann(1971) and Volz (1973). We have found that we can reproduce the full range of $\alpha$-values only by assuming that most of the extinction is produced by a water-soluble aerosol component characterized by a water-like refractive index and low absorption. Then, the different $\alpha$-values may be produced by the same material but different average aerosol sizes.

In figure 2 we demonstrate the effect of particle growth. We have plotted the evolution of a distribution of water-soluble particles within the $\alpha-k_{\mathrm{D}}(V)$ diagram. Two limiting cases are considered: number conservation according to a process of aerosol growth by condensation from the gas and mass conservation representative for a growth driven by aggregation of the aerosols. The lines mark the way of growth connecting the points of particles with neighbouring mean radii.

A comparison of this diagram with the $\alpha-k_{\mathrm{D}}(V)$ plot of the observations shows that the number conserving process reproduces the set of observations very well. Therefore, the different points of the observations may be caused by aerosols which have started with a similar value of the number density but have grown to different sizes due to their different history. 


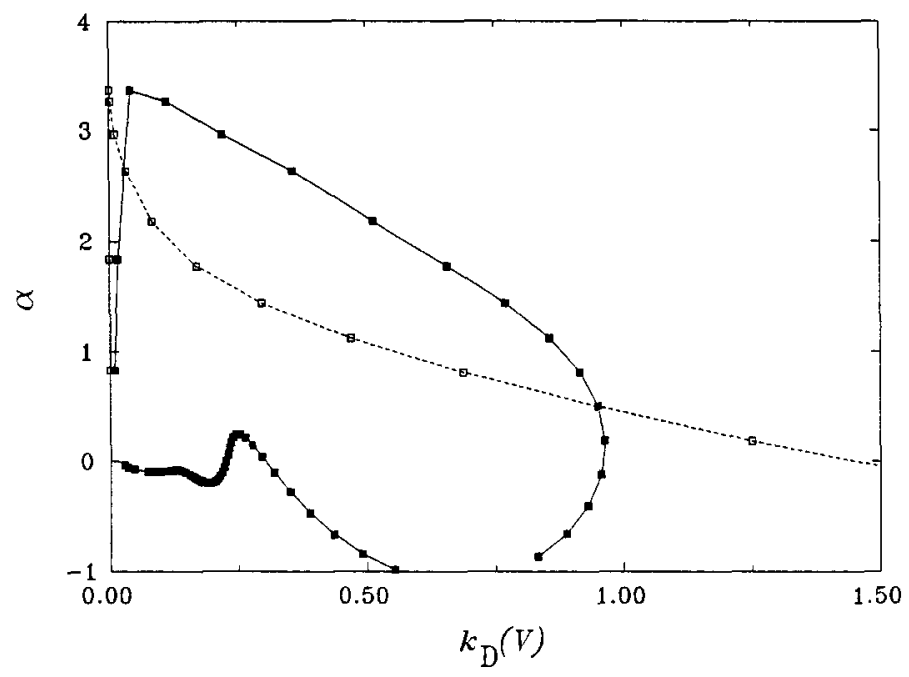

Figure 2 Wavelength exponent $\boldsymbol{\alpha}$ versus absolute aerosol extinction $k_{\mathrm{D}}(V)$. Evolution of water-soluble aerosols under mass conservation (filled squares) and under number conservation (open squares).

\section{Correlation with meteorological conditions}

Parallel weather observations at the meteorological station of the Jena University allowed us to find correlations between the variation of extinction parameters and the weather conditions.

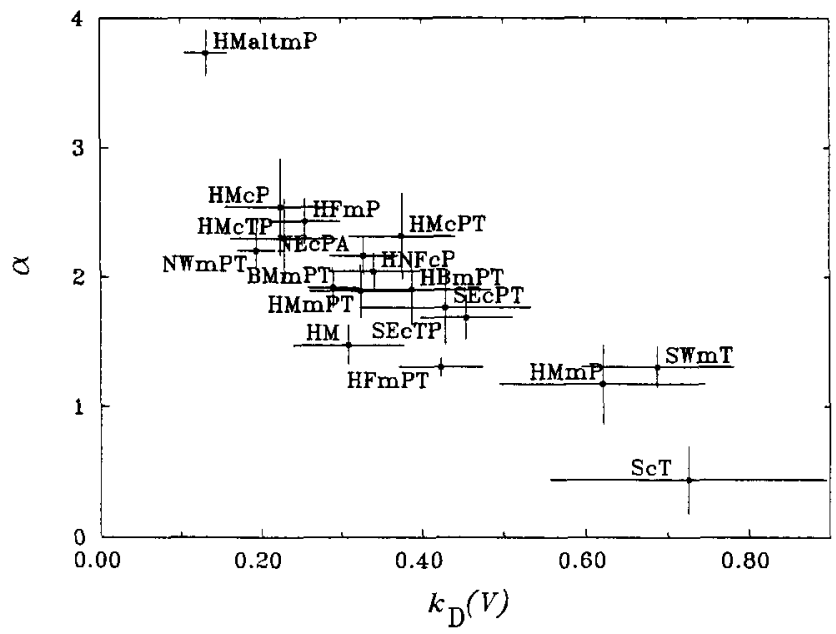

Figure 3 Mean $\alpha$ versus $k_{D}(V)$ values related via circulation types and air masses. Error bars are given. 
In the figure we present the mean $\alpha$ versus $k_{\mathrm{D}}(V)$ values for given meterological conditions. The meteorological conditions were classified by the atmospheric circulation type (see Heyer 1988) and the air-mass (see Critchfield 1960). The first capitalized letters of the abbreviations characterize the circulation type and the following letters denote the air-mass.

It is significant that there exist at least three different groups in the arrangement of the data points following the same tendency of decreasing $\alpha$ with increasing $k_{\mathrm{D}}(V)$. Although it is not possible to find a simple relation between extinction and meteorological conditions we found two general tendencies: Air-masses which come directly from higher latitudes (letter symbol on second position "P") contain smaller particles then those directly transported from lower latitudes (letter symbol on second position "T"). Best observing conditions occur in air-masses carrying low air-moisture (cTP, $\mathrm{cP}$, altmP) while more humid maritime air-masses produce high extinction and a low wavelength exponent $\alpha$.

\section{Nightly variations of the extinction}

Nightly time dependent extinction changes have been known for some times and are one of the main sources of errors in all-sky photometry (Angione 1984, Stickland et al. 1987).

In case of intermediate band and narrow band photometry where the deviations from a monochromatic behaviour are small and under the assumption that the extinction varies slowly and isotropically in the whole azimuth angle considered we replace the classical Bouguer line by

$$
m_{\mathrm{s}}-m_{\mathrm{obs}}=L\left(\lambda_{\circ}\right)-k\left(\lambda_{\circ}\right) X+D\left(\lambda_{\circ}\right) t .
$$

$m_{\mathrm{s}}$ means the standard magnitude of the star, $m_{\mathrm{obs}}$ the ground-based measurement at zenith distance $z, L\left(\lambda_{0}\right)$ the instrumental zero point at some photometric passband characterized by its mean wavelength $\lambda_{\circ}$ and $D\left(\lambda_{0}\right)$ represents a drift coefficient of extinction with time $t$ (Reimann et al. 1989). A statistical analyse shows that the visual drift coefficient can reach relatively high values whereas the drift coefficients in the colour indices remain generally low.

To get a plausible explanation for the observed drift coefficients in agreement with the results from the Mie calculations we related $D(y)$ to the absolute humidity of the air at the observing site in terms of the meteorological conditions (see figure 4). It seems significant that there exist at least two groups of different mechanism causing variable extinction.

Within the first group exhibiting characteristic low $D(y)$ values there exists a clear dependence that the drift coefficient grows with increasing moisture. This indicates that in this group the main process which is responsible for the nightly drift is the shrinking or swelling of the aerosols due to the water vapour content of the atmosphere at the observing site.

Separated from the first there exist a second group of meteorological conditions connected with high $D(y)$ values which does not seem to show any trend with mois- 
ture. In these cases the high values of the drift coefficient are caused by the exchange of different air-masses in higher layers over the observing site in a relatively short time interval.

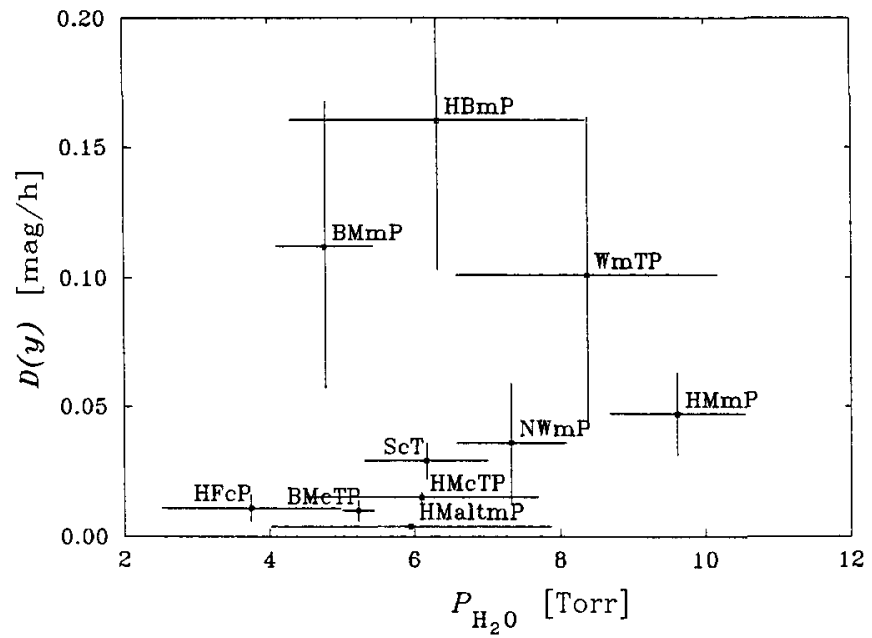

Figure 4 The drift coefficient versus water-vapour pressure diagram. Each point represents the mean value for the specified meteorological condition. Error bars are given.

\section{Longtime variations of extinction}

There is no doubt about the fact that the state of the atmosphere as well as its changes is influenced by anthropogeny activity contaminating the air with strange kinds of gaseous or dusty aerosols. But also natural catastrophes as large volcanic eruptions could induce long-time perturbations of the normal distribution of stratospheric aerosols. We found in our data a considerable increase of the visual extinction after the eruption of El Chichon in 1982 with the maximum in 1983. Taking the time interval since 1989 with definite lower extinction values as baseline interval characteristic for an unperturbed stratosphere we derived a maximum extinction excess caused by the El Chichon eruption of about $0.1 \mathrm{mag}$. This is in good agreement with estimated excess values for the sites of Gornergrat and Jungfraujoch between 0.06 and $0.1 \mathrm{mag}$ (Rufener 1986). Other important volcanic eruptions as e.g. the Mount St. Helens event are not traceable in our data.

Beside the normalization of the stratospheric aerosol content since the eruption of the $\mathrm{El} \mathrm{Chichon} \mathrm{there} \mathrm{seems} \mathrm{to} \mathrm{be} \mathrm{a} \mathrm{second} \mathrm{long-time} \mathrm{trend} \mathrm{present} \mathrm{in} \mathrm{our} \mathrm{data.}$ We found that the mean values of the measurements from the earliest to the latest observations has continuously shifted towards higher $\alpha$-values (see figure 1). This small growth of the mean $\alpha$ values since the classic measurements by Wempe 1947 is probably a result of a slow change of the general chemical constituents of the anthropogenic induced aerosols. 


\section{Conclusions}

The analysis of the visual drift coefficient $D(y)$ shows that the main part of variable nights is caused by swelling or shrinking aerosols due to the changing water vapour content in connection with certain meteorological conditions. As we have shown this is not only restricted to the lower tropospheric layers and, therefore, also conclusive for mountain observing stations. Even during clear weather conditions qualified as photometric we highly recommend frequent observations of standard stars to detect nightly variable extinction using Eq. (2).

An improved relation between meteorological conditions and extinction parameters as we have found could have predictive value for a better organization of different types of photometric observations and the reverse approach - the extinction correction of remote sensing images.

A theoretical explanation of the observed extinction data is possible when one assumes that the main part of the aerosol extinction is contributed by an aerosol with optical constants typical for the water-soluble type. Almost the full range of the observed points in the $\alpha-k_{\mathrm{D}}(V)$ diagram can be reproduced with nearly the same number density of scatterers but different sizes. This indicates condensation as the main process of aerosol growth.

Several analyses of volcanic produced aerosols (e.g. Cardelli \& Ackerman 1983) show that dust and ash aerosols are considerably larger than aerosols associated with gas-to-particle conversion. In analogy, we would interpret the long-time increase in the $\alpha$-values as a historical shift of the composition of the antropogeny atmospheric pollutants from the more dust/soot-like types to the gaseous types producing aerosols by gas-to-particle conversion. This requires, of course, further confirmation by direct aerosol measurements.

\section{References:}

Angione R.J., 1984, in: Proceedings of the workshop on improvements to photometry, NASA Conference Publication 2350, 1

Cardelli J.A., Ackerman T.P., 1983, PASP 95, 451

Critchfield H.J., 1960, General Climatology, Prentice-Hall, Inc., Englewood Cliffs, N.J.

Heyer E., 1988, Witterung und Klima: eine allgemeine Klimatologie, Teubner Verlagsgesellschaft, Leipzig

Koepke P., Hess M., 1988, Applied Optics 27, 2422

Reimann H.-G., Böhm M., Pfau W., 1989, Astron. Nachr. 310, 41

Rufener F., 1986, A\&A 165, 275

Stickland D.J., Lloyd C., Pike C.D., Walker N.E., 1987, The Observatory 107, 74

Twitty J.J., Weinman J.A., 1971, J. Appl. Meteor. 10, 725

Volz F., 1973, Appl. Optics 12, 564

Wempe J., 1947, Astron. Nachr. 275, 1 


\section{Discussion}

A. T. Young: The relation between humidity and aerosol optical properties has been very extensively investigated in the geophysics literature (see the review of G. Hamel, Adv. in Geophys. 19, 73 1976). The physical mechanism is understood: above 70\% relative humidity, salt particles dissolve and form large droplets, between $30 \%$ and $70 \%$ R.H., the biosols swell and shrink; there is little effect below 30\%. It is the relative rather than the absolute humidity that is important. This model has been used for 20 years or more in the AFGL atmosphere models LOWTRAN, MODTRAN, etc.

Reimann: The relative humidity of air-masses differs strongly between summer and winter months and therefore a general relation between drift coefficient and meteorological conditions via the relative humidity cannot be easily found.

B.Nicolet: A simple method to monitor the extinction coefficient $k_{\lambda}$ has been used for $\sim 30$ years, (Rufener). Two extinction stars, one ascending and one descending, are observed several times a night to deduce extra-atmospheric magnitudes and $k_{\lambda}$. Even during the best photometric nights, variations in $k_{\lambda}$ are observed; they mimic a phenomenon incorrectly interpreted as anisotropic extinction.

Reimann: We followed another method to detect time dependant extinction. We frequently observed a greater number of standard stars, carefully selected according to colour and air mass, in a solid azimuth angle. We have done this under the assumption that the extinction is isotropic. 